\title{
Consulta del viajero (II). Vacunas y profilaxis
}

\author{
A. García de Francisco, C. Prieto Zancudo
}

E.A.P. Alameda de Osuna I. Área 4 Atención Primaria. INSAM

\section{RESUMEN}

La indicación de vacunas y quimioprofilaxis a una persona que va a viajar a un país tropical, forma par te de la consulta al viajero antes de realizar el viaje.

Las vacunas de enfermedades típicamente tropi cales como la fiebre amarilla, encefalitis japonesa, etc., se indican según la zona a visitar y el riesgo de transmisión de la enfermedad.

Es importante resaltar que la mayoría de las en fermedades que forman parte del calendario vacu nal sistemático de los países occidentales (saram pión, polio, tétanos, etc.), son muy prevalentes en los países tropicales, por esta razón el viajero tiene que estar correctamente inmunizado frente a estas enfermedades antes de realizar el viaje.

Existen otras enfermedades que son poco fre cuentes en nuestro medio y que no están incluidas en el calendario vacunal sistemático (fiebre tifoi dea, hepatitis A, etc.), pero en países en vías de de sarrollo constituyen una importante causa de mor bimortalidad y por lo tanto está indicada la inmunización de éstas en viajeros.

La enfermedad más importante susceptible de quimioprofilaxis en países tropicales es el paludis mo. Es una enfermedad que puede producir la muerte al viajero y constituye una de las causas infecciosas de muerte más importantes en los paí ses en vías de desarrollo.

Haremos también una mención importante a la profilaxis de la diarrea del viajero por su alta fre-
Traveler's consultation (II). Vaccines and prophylaxis

\begin{abstract}
The indication of the vaccines and prophylaxis in travelers of the tropical countries, make up the traveller's consultation before the travel.

The vaccines of the typically tropical dise ases like yelow fever, japanesse encephalitis, etc., depends on the areas to visite and the risk of transmission in the differents tropical dise ases.

It's important to mention that the diseases that are included in the calendar of systematic vaccina tions in our countries (measles, poliomyelitis, teta nus, etc.), are very prevalents in the tropical coun tries, and this is the reason that the traveler must be correctly immunized from these diseases before the travel.
\end{abstract}

There are other diseases not very frecuent in our countries and they aren't included in the calendar of systematic vaccinations (typhoid fever, hepatitis A, and so on), but in the tropical coun tries are a very important cause of morbymorta lity and it is indicated the inmunization in the tra velers.

The most important disease capable of chemo prophylaxis in the tropical counties is the malaria. The malaria can produce death to travellers and it's one of the causes of death infectious more im portant in the tropical countries.

We will also do an important mention about the prophylaxis of the traveller's diarrhoea, because

Aceptación: 03-07-01 
cuencia de aparición cuando se realizan viajes in ternacionales.

Palabras clave: Vacunas. Profilaxis. Viajero. Tropical. it's very frecuent and prevalent in international travels.

Key words: Vaccines. Prophylaxis. Traveler. Tro pical.

\section{VACUNAS}

\section{Vacunas sometidas a reglamentación internacional}

\section{Fiebre amarilla}

El certificado de vacunación es exigido en muchos países a los viajeros que hayan estado en países endémicos (Tabla I). Se recomienda la vacunación a los viajeros que vayan a estos países. La vacuna es eficaz a partir de los 10 días posteriores a la administración (una dosis de $0,5 \mathrm{ml}$ vía subcutánea). Es una vacuna de virus vivos atenuados. Está contraindicada en niños menores de 1 año (en casos muy seleccionados ${ }^{1}$, se pueden vacunar a niños mayores de 4 meses), inmunodeprimidos graves, primer trimestre del embarazo y alérgicos al huevo y aminoglicósidos. Se remitirá al viajero a Sanidad Exterior (C/Francisco Silvela nº57, Madrid).

\section{Vacunas rutinarias (calendario vacunal)}

Es de suma importancia tener el calendario vacunal actualizado.

\section{Tétanos-difteria}

Administrada normalmente en la infancia. La dosis de recuerdo en el adulto debe administrarse cada diez años. Recomendada especialmente en aquellos viajeros con destinos en Argelia, Ecuador y países de la antigua Unión Soviética.

\section{Polio}

Los niños seguirán el calendario vacunal y los adultos que visiten zonas de riesgo (África subsahariana), recibirán una dosis de vacuna inactivada I.M. tipo Salk.

\section{Hepatitis B}

Recomendada a todos los viajeros no inmunizados, especialmente a los que vayan a trabajar en puestos sanitarios y a los que tengan intención de realizar turismo sexual. Existe una pauta clásica de administración de 3 dosis en los meses 0-1-6 y otras dos ultrarápidas igual de eficaces de 0-1-2 meses y 0-15-30 días².

\section{Triple vírica (sarampión, paperas y rubéola)}

Se recomienda en cooperantes sanitarios que no estén inmunizados. Está contraindicada en personas con alergia a neomicina. Se aconseja posponer el embarazo 3 meses después de haber recibido la vacuna.

\section{Varicela}

Recomendada en viajeros que vayan a tener contacto estrecho con niños (escuelas, orfanatos, hospitales infantiles). Está contraindicada en inmunodeprimidos y embarazadas, y de forma relativa en niños que hayan realizado de forma reciente tratamiento con aspirina por el riesgo de síndrome de Reye ${ }^{3}$.

\section{Neumococo}

Recomendada en adultos mayores de 65 años y en viajeros con problemas cardiopulmonares y enfermedades crónicas. Se administra una sola dosis de vacuna con un recuerdo a los 5 años.

\section{Gripe}

Recomendada en los mismos supuestos que la vacuna antineumocócica.

\section{Haemophilus influenzae}

Contraindicada en esplenectomizados, por riesgo de septicemia.

\section{Vacunas recomendadas y en situaciones especiales}

\section{Hepatitis A}

Recomendada a todos los viajeros no inmunizados, que visiten países tropicales y subtropicales. Consta de 3 dosis 0, 1, 12 meses. Existe una vacuna combinada de hepatitis A y B (Twinrix $\left.{ }^{\circledR}\right)^{4}$, con 
PAÍSES ENDÉMICOS DE FIEBRE AMARILLA

\section{África}

Angola

Provincias: Bengo y Luanda

Benin

Departmento: Atakora

Burkina Faso

Region Gaoua

Camerún

Provincia Northern

República Democrática del Congo

$10^{\circ}$ al Norte y Sur del país

Gabón

Provincia de Ogooue'-Ivindo

Gambia

División Upper River

Ghana

Región Upper West

Guinea

Región Siguiri

Liberia

Boma County, Bong County, Bassa County, Sinoe County

Nigeria

Estados: Anambra, Bauchi, Bendel, Benue, Cross River, Imo, Kaduna, Kano, Kwara, Lagos, Niger, Ogun, Ondo, Oyo y Plateau.

Sierra Leona

Distrito de Kenema

Sudán

Al Sur de $12^{\circ}$ al Norte

América del Sur

\begin{tabular}{|c|c|}
\hline Bolivia & Departmentos: Beni, Cochabamba, La Paz y Santa Cruz. \\
\hline Brasil & $\begin{array}{l}\text { Estados: Amapá, Amazonas, Bahia, Coribe, Goiás, Maranhão, Mato Grosso, } \\
\text { Minas Gerais, Pará, Roraima, São Paulo y Tocantins }\end{array}$ \\
\hline Colombia & $\begin{array}{l}\text { Departamentos: Antioquia, Boyaca, Cesar, Choco, Cundinamarca, Norte de } \\
\text { Santander y Vichada. }\end{array}$ \\
\hline Ecuador & $\begin{array}{l}\text { Intendencias: Arauca, Caqueta, Casanare, Cucuta, Guaviare, Meta y Putumayo. } \\
\text { Provincias: Morona-Santiago, Napo, Orellana, Pastaza, Sucumbios y Zamora } \\
\text { Chinchipe. }\end{array}$ \\
\hline Guayana Francesa & Region de Saint Laurent-du-M oroni \\
\hline Perú & $\begin{array}{l}\text { Departamentos: Amazonas, Ancash, Ayacucho, Cusco, Huánuco, Junin, Loreto, } \\
\text { Madre de Dios, Puno, Pasco, San Martin y Ucayali. }\end{array}$ \\
\hline Venezuela & Estado de Amazonas y Bolivar. \\
\hline
\end{tabular}

http:/ / www.cdc.gov/ travel/ diseases.htm

la misma pauta de administración que la vacuna de la hepatitis $\mathrm{B}$.

\section{Fiebre tifoidea}

La vacuna oral (Vivotif-Berna®), está compuesta de bacterias vivas atenuadas. Consta de 3 dosis y la pauta es una dosis cada $48 \mathrm{~h}$; se toma antes de realizar el viaje (3 semanas antes), ya que empieza a ser efectiva a partir de 2 semanas después de la última dosis. No se aconseja administrarla junto con la Polio oral, tampoco se recomienda administrarla junto con antibióticos o medicación para profilaxis de la mala- ria, hasta después de haber transcurrido una semana de la última dosis de la vacuna oral ${ }^{1}$. No se indica en inmunodeprimidos, embarazadas y niños menores de 6 años. La vacuna parenteral (Typhim Vi-Pasteur Merieux Connaught $\left.{ }^{\circledR}\right)$, es una vacuna de polisacáridos capsulares y carece de las contraindicaciones de la vacuna de bacterias vivas. Se puede administrar en adultos y niños a partir de 2 años.

\section{Meninigitis}

En España está comercializa la bivalente A-C. Se recomienda en turistas adultos y niños no vacunados 
que viajen a zonas donde se detectan brotes epidémicos frecuentes (África Subsahariana, Oriente Medio, India, Nepal, Sudeste asiático, zonas de Brasil y las pequeñas antillas del Caribe). También recomendada en viajeros peregrinos a la $\mathrm{Meca}^{6}$. Se administra vía subcutánea y es eficaz a partir de las 2 semanas, con una duración aproximada de la inmunidad de 3 años.

\section{Cólera}

La vacuna sólo se indica a cooperantes que vayan a trabajar a zonas donde existan epidemias declaradas o a viajeros que visiten esas zonas durante el viaje.

\section{Rabia}

Se aconseja vacunar a viajeros de riesgo (cazadores, veterinarios, biólogos, y personal logístico cooperante). Se administran 3 dosis intramusculares, los días 0,7 y 21 ó 28 antes del viaje. Los laboratorios que la tienen comercializada son: Pasteur Merieux Connaught (Imovax $\left.{ }^{\circledR}\right)$, Smithkline Beecham (RVA®) y Avert-Chiron (Rab®). Solamente la Imovax ${ }^{\circledR}$, puede administrarse vía intradérmica. La producción de anticuerpos se ve disminuida si el viajero toma simultáneamente cloroquina, especialmente cuando la vacuna es administrada vía intradérmica.

\section{Encefalitis primavero-estival}

Indicada a viajeros de aventuras que vayan a visitar zonas rurales de Rusia, República Checa, Eslovenia, Alemania, Finlandia y Austria. Es una vacuna de virus vivos inactivados y se administra en 3 dosis i.m. a los 0-1-12 meses. No está contraindicada en el embarazo.

\section{Encefalitis japonesa}

Se recomienda solamente a personas que vayan a trabajar o realicen estancias prolongadas en las zonas rurales de China, Corea, subcontinente indio, sudeste asiático, Japón, Taiwan, Singapur, Hong Kong y este de Rusia. Se administra vía subcutánea en 3 dosis los días 0-7-30, preferiblemente 2-4 semanas antes del viaje y se aconseja repetir una dosis a los 3 años, si se vuelve a indicar al mismo viajero. Nombres comerciales JE-Vax® (Pasteur Merieux Connaught).

\section{Enfermedad de Lyme}

Recomendada a individuos entre 15-70 años que viajen a zonas endémicas (Noreste, Medioeste y costa del Pacífico de EE.UU.). La pauta es de 0-112 meses vía intramuscular en la región deltoidea, se recomiendan extremar las medidas profilácticas, ya que la vacuna no confiere una protección total.

\section{PROFILAXIS PARA LA MALARIA}

La malaria o paludismo es la principal parasitosis del ser humano. Afecta a unos 2.500 millones de personas en más de 100 países del mundo ${ }^{7}$.

Los CDC (Centers Diseases Control) americanos, disponen de una información muy actualizada sobre la situación de la malaria en el mundo, tienen además una página web a disposición de todos los usuarios de la red www.cdc.gov/travel/ blusheet.htm, para todos aquellos viajeros que vayan a visitar un país infectado por la malaria.

La profilaxis se realiza según la zona y país que se vaya a visitar (Tabla II). Antes de indicar a un viajero la profilaxis es muy importante valorar los siguientes condicionantes:

1. Duración del viaje.

2. Antecedentes médicos del viajero ${ }^{8}$.

3. Tipo de viaje: bajo riesgo (estancias cortas en zonas urbanas, como en los viajes de negocios, congresos) y alto riesgo (estancias prolongadas en zonas rurales, como el viaje de cooperante sanitario).

4. Lugares que se vaya a visitar: ciudad, selva o zonas rurales.

5. Estación climatológica: la estación de lluvias favorece más la transmisión de la enfermedad.

6. Altitud: es muy raro observar el mosquito Anopheles (vector de la enfermedad) en altitudes superiores a $2.000 \mathrm{~m}^{9}$.

La quimioprofilaxis para la malaria se suele realizar una semana antes del viaje, durante el viaje y 4 semanas después del viaje ${ }^{10}$.

Entre los distintos medicamentos quimioprofilácticos se encuentran:

-Proguanil (Paludrine®) que se ha de tomar en dosis diaria de $200 \mathrm{mg}$ ( 2 comprimidos de $100 \mathrm{mg}$ al día). Se administra en pauta conjunta con la cloroquina como coadyuvante. Generalmente es bien tolerado y presenta escasos efectos secundarios. El proguanil se puede utilizar en pediatría y en el embarazo $^{11}$.

-Doxiciclina (Vibracina ${ }^{\circledR)}$ también se ha de administrar diariamente en dosis $100 \mathrm{mg}$ (1 comprimido al día). Recomendada en viajes a regiones donde existe resistencia del Plasmodium a la cloroquina y mefloquina. Su administración debe realizarse con alimentos, ya que de forma ocasional puede producir úlcera esofágica. En pautas muy prolongadas (años), puede causar alteraciones dérmicas y fotosensibilidad, y en raras ocasiones necrosis hepática. Está contraindicada en todo el embarazo. 
RIESGO POR ÁREAS GEOGRÁFICAS EN PAÍSES CON MALARIA ENDÉMICA

\begin{tabular}{lll}
\hline País & Áreas de riesgo dentro del país & Profilaxis recomendada \\
\hline Afghanistan & Todo & Mefloquina \\
\hline Argelia & Muy limitado a la región del Sahara & Ninguna \\
\hline Angola & Todo & Mefloquina \\
\hline Argentina & P vivax en áreas rurales fronterizas con Bolivia y Paraguay & Cloroquina \\
\hline Armenia & Riesgo limitado al oeste de las zonas fronterizas: Masis, Ararat y & Cloroquina \\
\hline Azerbaiyan & Artashat regiones en el distrito de Ararat. No riesgo en zonas turistas. & Cloroquina \\
\hline Bangladesh & Áreas de la frontera Sur y Norte de la región de Khachmas & Mefloquina \\
\hline Belize & Todo. No riesgo en la ciudad de Dhaka & Cloroquina \\
\hline Benin & $\begin{array}{l}\text { Áreas rurales incluyendo las playas de las islas y zonas forestales } \\
\text { conservadas. No riesgo en la costa central del distrito de Belize. }\end{array}$ & Mefloquina \\
\hline Bhutan & Todo & Mefloquina \\
\hline Bolivia & Áreas rurales en los distritios fronterizos con la India & Mefloquina \\
& $\begin{array}{l}\text { Áreas rurales < 2.500 metros solamente, excepto en el departamento } \\
\text { de Oruro y Provincia de Ingavi, Los Andes, Omasuyos, Pacajes, Sur } \\
\text { y zona Centro del departamento de Potosi donde no hay riesgo. }\end{array}$ &
\end{tabular}

\begin{tabular}{|c|c|c|}
\hline Botswana & Norte del país (Norte de $21^{\circ}$ Sur) desde noviembre a junio & Mefloquina \\
\hline Brasil & $\begin{array}{l}\text { Riesgo en Acre y Rondonia, territorios de Amapa y Roraima, y } \\
\text { en areas rurales de Amazonas, Maranhao, Mato Grosso, Para y } \\
\text { Tocantins. Las afueras de Manaos y Porto Velho son áreas de riesgo. } \\
\text { Nota: No hay riesgo para viajeros en la costa de los estados que } \\
\text { forman un cuerno hasta la frontera de Uruguay y cataratas de Iguazú. }\end{array}$ & Mefloquina \\
\hline Burkina Faso & Todo & Mefloquina \\
\hline Burundi & Todo & Mefloquina \\
\hline Camboya & $\begin{array}{l}\text { Todo, excepto en Phnom Penh y alrededores del Tonle Sap. } \\
\text { Existe riesgo de malaria en Angkor Wat. }\end{array}$ & $\begin{array}{l}\text { Mefloquina (doxiciclina } \\
\text { en el oeste fronterizo) }\end{array}$ \\
\hline Camerún & Todo & Mefloquina \\
\hline Cabo Verde & $\begin{array}{l}\text { Existe un riesgo limitado en las islas de Sao Tiago desde } \\
\text { septiembre a noviembre }\end{array}$ & Ninguna \\
\hline
\end{tabular}

\begin{tabular}{|c|c|c|}
\hline $\begin{array}{l}\text { República } \\
\text { Centroafricana }\end{array}$ & Todo & Mefloquina \\
\hline Chad & Todo & Mefloquina \\
\hline China & $\begin{array}{l}\text { Áreas rurales solamente en: Anhui, Fujian, Guangdong, Guangxi, } \\
\text { Guizhou, Hainan, Hubei, Hunan, Jiangsu, Jiangxi, Shandong, } \\
\text { Shanghai, Sichuan, Xinjiang, Xizang, Yunnan y Zhejiang } \\
\text { provincias/ regiones autónomas. La transmisión ocurre }<1.500 \text { metros } \\
\text { desde julio a noviembre } 33^{\circ} \text { de Norte a Norte, de mayo a diciembre } \\
\text { entre } 33^{\circ} \text { Norte y } 25^{\circ} \text { Norte y siguiendo el año por debajo de } 25^{\circ} \text { Norte. } \\
\text { Nota: Viajeros que visiten ciudades y zonas populares turísticas } \\
\text { no requieren profilaxis. }\end{array}$ & $\begin{array}{l}\text { Cloroquina (mefloquina } \\
\text { para Hainan Islas y Sur } \\
\text { de provincias } \\
\text { fronterizas con } \\
\text { Myanmar, Laos y } \\
\text { Vietnam) }\end{array}$ \\
\hline Colombia & $\begin{array}{l}\text { En general, áreas rurales solamente, no hay riesgo en Bogota } \\
\text { y los alrededores }\end{array}$ & Mefloquina \\
\hline Comoros & Todo & Mefloquina \\
\hline Congo & Todo & Mefloquina \\
\hline
\end{tabular}


RIESGO POR ÁREAS GEOGRÁFICAS EN PAÍSES CON MALARIA ENDÉMICA (continuación)

\begin{tabular}{lll} 
País & Áreas de riesgo dentro del país & Profilaxis recomendada \\
\hline Costa Rica & $\begin{array}{l}\text { Áreas rurales solamente (incluyendo áreas turistas). No hay riesgo } \\
\text { en la zona central de las tierras altas. Riesgo limitado en áreas } \\
\text { rurales de Alajuela, Guanacaste, Limon, Heredia y provincia de } \\
\text { Los Chiles. }\end{array}$ & Cloroquina \\
\hline
\end{tabular}

\begin{tabular}{llr}
\hline Côsta de Marfil & Todo & Mefloquina \\
\hline República & Todo & Mefloquina
\end{tabular}

Democrática

del Congo

\begin{tabular}{lll}
\hline Djibouti & Todo & Mefloquina \\
\hline República & Todas las áreas rurales. Alto riesgo en zonas fronterizas con Haiti. & Cloroquina \\
Dominicana & Los viajeros que visiten zonas turísticas no requieren profilaxis & \\
\hline
\end{tabular}

Ecuador Todas las provincias a lo largo de la frontera este y costa del Mefloquina

Pacífico: Canar, Cotopasi, El Oro, Esmeraldas, Guayas, Los Rios,

Manabi, M orona-Santiago, Napo, Pastaza, Pinchincha, Sucumbios,

Zamora, Chinchipe. (No hay riesgo en Quito y alrededores,

zona central de las altas tierras turísticas e Islas Galápagos)

\begin{tabular}{|c|c|c|}
\hline Egipto & $\begin{array}{l}\text { Riesgo limitado en El Faiyum y parte del Sur (Alto Egipto). } \\
\text { No hay riesgo en las zonas turísticas (incluyendo los cruceros) }\end{array}$ & Cloroquina \\
\hline El Salvador & Solamente en áreas rurales & Cloroquina \\
\hline Guinea Ecuatorial & Todo & Mefloquina \\
\hline Eritrea & Todo. No hay riesgo en Asmara y a partir de 2.000 metros & Mefloquina \\
\hline Ethiopia & Todo, excepto en Addis Ababa y a partir de 2.000 metros & Mefloquina \\
\hline Guayana Francesa & Todo & Mefloquina \\
\hline Gabon & Todo & Mefloquina \\
\hline Gambia & Todo & Mefloquina \\
\hline Ghana & Todo & Mefloquina \\
\hline Guatemala & $\begin{array}{l}\text { Sólo zonas rurales. No hay riesgo en alturas }>1.500 \text { metros } \\
\text { en la zona central }\end{array}$ & Cloroquina \\
\hline Guinea & Todo & Mefloquina \\
\hline Guinea-Bissau & Todo & Mefloquina \\
\hline Guyana & $\begin{array}{l}\text { Alto riesgo en áreas rurales de las regiones del interior } \\
\text { incluyendo Rupununi, regiones del Noroeste y a lo largo del } \\
\text { Río Pomeroon. El riesgo en Georgetown y Nueva Amsterdam es bajo. }\end{array}$ & Mefloquina \\
\hline Haiti & Todo & Cloroquina \\
\hline Honduras & Alto riesgo de $\mathrm{P}$. vivax en áreas rurales solamente & Cloroquina \\
\hline India & $\begin{array}{l}\text { Todas la áreas por debajo de } 2.000 \text { metros incluyendo Delhi y Bombay. } \\
\text { No hay riesgo en parte de los estados de Himachal Pradesh, } \\
\text { Jammu, Kashmir y Sikkim }\end{array}$ & Mefloquina \\
\hline Indonesia & $\begin{array}{l}\text { En general, solamente en áreas rurales, excepto alto riesgo en todas } \\
\text { las áreas de Irian Jaya (mitad oeste de la isla de Nueva Guinea). } \\
\text { No hay riesgo en ciudades de Java y Sumatra y alrededores de Java o } \\
\text { Bali. Nota: existe transmision en áreas rurales no visitadas por turistas. }\end{array}$ & M efloquina \\
\hline
\end{tabular}




\section{RIESGO POR ÁREAS GEOGRÁFICAS EN PAÍSES CON MALARIA ENDÉMICA (continuación)}

\begin{tabular}{llc} 
País & Áreas de riesgo dentro del país & Profilaxis recomendada \\
\hline $\begin{array}{l}\text { República } \\
\text { Islámica de Irán }\end{array}$ & $\begin{array}{l}\text { Riesgo limitado a áreas rurales solamente (marzo a noviembre) en las } \\
\text { provincias de Sistan-Baluchestan, Kermany y Hormozgan, el sur de Fars, } \\
\text { Kohgiluyeh-Boyar, Lorestan and Chahar Mahai-Bakhtiani y el norte de Khuzestan. }\end{array}$ & M efloquina \\
\hline
\end{tabular}

\begin{tabular}{ll}
\hline Iraq Todas las áreas del norte (mayo a noviembre): Duhok, Erbil, Basrah, Cloroquina \\
Tamim, Ninawa y provincia de Sulaimaniya.
\end{tabular}

\begin{tabular}{lll}
\hline Kenya & Todas las áreas incluyendo los parques nacionales. Bajo riesgo en & Mefloquina \\
& la ciudad de Nairobi y por encima de 2.500 metros
\end{tabular}

\begin{tabular}{lll}
\hline Korea del Norte & Riesgo de P. vivax en algunas zonas del Sur. & Ninguna \\
\hline Korea del Sur & $\begin{array}{l}\text { Riesgo de P. vivax a lo largo de la zona desmilitarizada (zonas no } \\
\text { visitadas por turistas). No se aconsejan antimaláricos a turistas. }\end{array}$ & Ninguna
\end{tabular}

\begin{tabular}{|c|c|c|}
\hline Laos & Todas las áreas, excepto en la ciudad de Vientiane & Mefloquina \\
\hline Liberia & Todo & Mefloquina \\
\hline Libia & $\begin{array}{l}\text { Riesgo limitado a dos pequeños focos en el suroeste del país de } \\
\text { febrero a agosto }\end{array}$ & Ninguna \\
\hline Madagascar & Todo & Mefloquina \\
\hline Malawi & Todo & Mefloquina \\
\hline Malaysia & $\begin{array}{l}\text { Áreas rurales remotas de la península y Sarawak (NW Borneo), } \\
\text { las zonas urbanas y la costa están libres de malaria. } \\
\text { Todas las áreas de Sabah (NE Borneo). }\end{array}$ & Mefloquina \\
\hline
\end{tabular}

\begin{tabular}{lll}
\hline Mali & Todo & Mefloquina \\
\hline Mauritania & $\begin{array}{l}\text { Todas las áreas excepto en el norte de Dakhlet-Nouadhibou y } \\
\text { Tiris-Zemour. En Inchiri y Adrar, el riesgo va de julio a octubre. }\end{array}$ & Mefloquina \\
\cline { 2 - 3 } & $\begin{array}{l}\text { Riesgo de P. vivax en áreas rurales solamente. } \\
\text { No hay riesgo en Islas Rodrigues }\end{array}$ & Cloroquina \\
\hline
\end{tabular}

\begin{tabular}{lll}
\hline Mayotte & Todo & Mefloquina \\
\hline Mexico & Áreas rurales de los siguientes estados: Campeche, Chiapas, & Cloroquina \\
& Chihauhua, Durango, Guerrero, Hidalgo, Jalisio, Michoacan, Nayarit, & \\
& Oaxaca, Quintana Roo, Sinaloa, Sonora y Veracrus.
\end{tabular}

\begin{tabular}{llc}
\hline Marruecos & Riesgo muy limitado de P. vivax en algunas zonas rurales & Ninguna \\
\hline Mozambique & Todo & Mefloquina \\
\hline Myanmar & Áreas rurales. Nota: viajeros a Yangon (Rangoon) y Mandalay & Mefloquina (doxiciclina \\
(Birmania) & no necesitan profilaxis. & en la frontera Tailandesa) \\
\hline Namibia & Todas las áreas de Ovamboland y Caprivi Strip & Mefloquina \\
\hline Nepal & Áreas rurales en el distrito de Terai y todos los distritos & Mefloquina \\
\hline
\end{tabular}

Nuevas Hebridas (ver Vanuatu)

\begin{tabular}{lll}
\hline Nicaragua & Áreas rurales y afueras de Bluefields, Bonanza, Chinandega, Leon, & Cloroquina \\
& Puerto Cabeza, Rosita y Siuna
\end{tabular}


Tabla II

RIESGO POR ÁREAS GEOGRÁFICAS EN PAÍSES CON MALARIA ENDÉMICA (continuación)

\begin{tabular}{lll} 
País & Áreas de riesgo dentro del país & Profilaxis recomendada \\
\hline Niger & Todo & Mefloquina \\
\hline Nigeria & Todo & Mefloquina \\
\hline Oman & Todo & Mefloquina \\
\hline Pakistan & Todas las áreas <2.000 metros incluyendo ciudades & Mefloquina \\
\hline Panama & Áreas rurales del norte y oeste del Canal & Cloroquina \\
& Áreas rurales del sur y este del Canal & Mefloquina \\
\hline Papua Nueva & No hay riesgo en la zona del Canal ni en Ciudad de Panama & \\
Guinea & Todo & Mefloquina \\
\hline Paraguay & Riesgo de P.Vivax en la frontera con Brasil & \\
\hline Perú & Sólo en áreas rurales. & Cloroquina \\
& Nota: no hay riesgo a viajeros que visiten Lima y alrededores, & Cloroquina \\
& costas del sur de Lima y altas tierras turísticas (Cuzco, Machu Picchu, & Mefloquina en la \\
& Lago Titicaca). & y Ecuador \\
\hline Filipinas & Sólo áreas rurales. En Manila y provincia de Bohol, Catanduanes & Cloroquina \\
& y Cebu no hacer profilaxis. & Mefloquina en zonas \\
& Resistencia a cloroquina en áreas rurales de Luzon, Basilian, Mindoro, & con resistencia a \\
& Palawan, M indanao y Sulu-Archipiélago. & cloroquina. \\
\hline Rwáncipe & Todo & Mefloquina \\
\hline Tanto Tome y & Todo & Mefloquina \\
\hline
\end{tabular}

Arabia Saudi Todas las áreas de las provincias de oeste, excepto en altas M efloquina altitudes de la provincia de Asir (frontera conYemen), y áreas urbanas de Jeddah, Meca, Medina y Taif

\begin{tabular}{lll}
\hline Senegal & Todo & Mefloquina \\
\hline Sierra Leona & Todo & Mefloquina \\
\hline Salomon Islas & Todo & Mefloquina \\
\hline Somalia & Todo & Mefloquina
\end{tabular}

Sudáfrica Áreas rurales (incluyendo parques nacionales) en el norte, este y oeste en áreas de baja altitud del Transvaal y en la Costa norte de Natal de $28^{\circ}$ sur.

Mefloquina

\begin{tabular}{lll}
\hline Sri Lanka & Todas las áreas rurales excepto en Colombo, Kalutara, Nuwara y Eliya. & Mefloquina \\
\hline Sudan & Todo & Mefloquina \\
\hline Surinam & $\begin{array}{l}\text { Sólo áreas rurales, excepto en Paramaribo distrito y costa norte a } \\
\text { partir de } 5{ }^{\circ} \text { Norte. }\end{array}$ & Mefloquina
\end{tabular}

\begin{tabular}{lll}
\hline Swaziland & Todas las tierras bajas & Mefloquina \\
\hline Siria & $\begin{array}{l}\text { Sólo áreas rurales (mayo a octubre) especialmente a lo largo } \\
\text { de la frontera norte. No hay riesgo en los distritos de Damasco, } \\
\text { Deir-es-zor y Sweida. }\end{array}$ & Cloroquina \\
&
\end{tabular}

Tajikistan

Riesgo predominante en el sur fronterizo (región de Khatlon); en zona

Cloroquina central (Dushanbe), oeste (Gorno-Badakhshan) y norte (Leninabad). Resistencia a cloroquina en algunas zonas. 
Tabla II

RIESGO POR ÁREAS GEOGRÁFICAS EN PAÍSES CON MALARIA ENDÉMICA (continuación)

\begin{tabular}{lll} 
País & Áreas de riesgo dentro del país & Profilaxis recomendada \\
\hline Tanzania & Todo & Mefloquina \\
\hline Tailandia & $\begin{array}{ll}\text { Riesgo de malaria en zonas rurales del país no visitadas por turistas. } \\
\text { No hay riesgo en ciudades y zonas de turismo (e.j. Bangkok, } \\
\text { Chiangmai, Pattaya, Phuket, Samui). } \\
\text { Resistencia a mefloquina en la frontera con Birmania y Camboya. }\end{array}$ & $\begin{array}{l}\text { Doxiclina en áreas } \\
\text { fronterizas con Camboya }\end{array}$ \\
\hline
\end{tabular}

\begin{tabular}{lll}
\hline Togo & Todo & Mefloquina \\
\hline Turquia & $\begin{array}{l}\text { Áreas de Cukurova/ Amikova y sudeste de Anatolia (abril a octubre). } \\
\text { No hay riesgo en zonas de turismo. }\end{array}$ & Cloroquina \\
& Todo & \\
\hline
\end{tabular}

\begin{tabular}{lll}
\hline Uganda & Todo & Mefloquina \\
\hline $\begin{array}{l}\text { Emiratos } \\
\text { árabes Unidos }\end{array}$ & $\begin{array}{l}\text { Riesgo en regiones rurales del norte de los Emiratos. No hay riesgo } \\
\text { en ciudades como Dubai, Sharjah, Ajman, Umm al Qaiwain y Emirato } \\
\text { de Abu Dhabi. }\end{array}$ & Cloroquina \\
\hline
\end{tabular}

\begin{tabular}{llc}
\hline Vanuatu & Todo, excepto en Isla Futuna. & Mefloquina \\
\hline Venezuela & $\begin{array}{l}\text { Sólo zonas rurales en toda la frontera de los estados y territorios } \\
\text { de Barinas, Merida y Portugesa }\end{array}$ & Mefloquina \\
\hline Vietnam & $\begin{array}{l}\text { Sólo áreas rurales. No hay riesgo en el Delta del Río Rojo y la costa } \\
\text { y meseta norte de Nha Trang. }\end{array}$ & Mefloquina \\
\hline Yemen & Todo excepto en Aden y áreas de aeropuertos. & Mefloquina \\
\hline Zambia & Todo & Mefloquina \\
\hline Zimbabwe & Todo excepto en ciudades como Harare y Bulawayo. & Mefloquina
\end{tabular}

Los países no listados se consideran libres de malaria.

† Adaptado del CDC Health Information for International Travel 1999-2000 and WHO International Travel and Health 2000.

-Cloroquina (Resochin $\left.{ }^{\circledR}\right)$ se toma una vez por semana, preferiblemente con alimentos. La dosis semanal de cloroquina, es de $300 \mathrm{mg}$ de cloroquina-base, que equivale a 2 comprimidos de $250 \mathrm{mg}$. Presenta escasos efectos adversos, de forma ocasional y al inicio del tratamiento puede producir náuseas y cefalea. Los efectos tóxicos retinianos sólo ocurren cuando se administra durante un largo periodo de tiempo en dosis diaria mayor de $100 \mathrm{mg}$. En la actualidad existe una presentación farmacológica conjunta de cloroquina y proguanil (Savarine $\left.{ }^{\circledR}\right)$, disponible en nuestro país. Este fármaco no está indicado en personas con antecedentes personales de epilepsia y en psoriasis generalizada. Se puede utilizar en pediatría y en el embarazo.

-Mefloquina (Lariam ${ }^{\circledR}$ ) se administra una vez por semana también y su dosis es de 1 comprimido de $250 \mathrm{mg}$ a la semana. Constituye el fármaco de elección en aquellas regiones donde el Plasmodium es resistente a la cloroquina ${ }^{12}$. No está indicada en menores de 1 año. Aproximadamente el 20-30\% de los viajeros que realizan profilaxis con este fármaco experimentan efectos secundarios, como náuseas, insomnio, pesadillas, cambios en el humor y diarrea. Tan sólo 1/10.000 individuos presentan reacciones neuropsiquiátricas severas (convulsiones, psicosis); por lo que no se recomienda en personas con antecedentes familiares y personales de epilepsia, trastornos psiquiátricos y en aquellas otras con alteraciones del equilibrio o alpinistas ${ }^{13}$.

-Actualmente hay nuevos fármacos en el mercado aún no comercializados en nuestro país ${ }^{14}$, como es la combinación de atovaquone/proguanil (Malarone $®)$, que ha demostrado gran eficacia en casos de infección por Plasmodium multirresistente, con excelente tolerancia y mínimos efectos secundarios. 
Otros fármacos en investigación son los derivados de la artemisa, azitromicina y la combinación de primaquina/tafenoquina, pero no existe suficiente evidencia para ser recomendados como antipalúdicos en la actualidad.
CORRESPONDENCIA:

Carmelo Prieto Zancudo

C/ Cobos de Segovia n ${ }^{\circ}$, portal $4,5^{\circ}$ A.

28005 Madrid

e-mail: cprietoz@nexo.es

\section{Bibliografía}

1. Capello M. Immunizations for children traveling abroad. Pediatr Infect Dis J 1998; 17 (2): 157-8. (Pr).

2. Jong EC. TA. Travel immunizations. Clin Med North Am 1999; 83 (4): 903-22. (Pr)

3. WHO. International Travel and Health. Vaccination Requeriments and Health Advice. Ginebra: OMS, 1996. (Ce).

4. Mitka M. Travelers need hepatitis vaccine and other immunizations. JAMA 2000; 283 (17): 2226-7. (Pr).

5. Advice for travelers. Med Lett Drugs Ther 1998; 40 (1025); 47-51. (Ce).

6. The yellow book. Health information for international Travel. Atlanta, Georgia: CDC, 1999. (Ce).

7. Ellis CJ. On achieving consensus on the prevention of malaria. J Antimicrob Chemother 1998; 41: 4-6. (Pr).

8. Valerio Ll, Roca C, Llorens MJ, Mascort JJ, Solsona LL, Ferrer-Vidal D. Profilaxis antipalúdica: el papel del médico de cabecera. Aten Primaria 1997; 47: 53-60.

9. Weiss EA. Medical considerations for wilderness and adventure travellers. Med Clin North Am 1999: 83 (4): 885-903. (Pr).
10. Croft A. Malaria prevention in travellers. BMJ 2000; 321 : 154-60. (Pr).

11. MacLeod C. La viajera embarazada. Clin Med Norteam 1992; 6: 1361-74. (Pr)

12. Handschin J. Mefloquine to prevent malaria: a systematic review of trials. BMJ 1997; 315: 1412-6. (Pr).

13. Botella de Maglia J. The role of the expediotionary physician. Med Clin Barc 1996; 107 (7): 270-4. (Pr).

14. Canadian guidelines for prevention and treatment of malaria. Appendix I: Malaria risk by geographic area in countries with endemic malaria. Canada Communicable Report Supplement. Vol. 2652 March 2000. (Ce).

Nota: abreviaturas de los tipos de estudios de las referencias blibliográficas recogidas, según el grado de evidencia científica:

Pr: Revisión clínica

$\mathrm{X}$ : Estudios transversales

Ce: Comité de expertos

Ra: Ensayo clínico aleatorio randomizado. 